\title{
Reduction of pp32 expression in poorly differentiated pancreatic ductal adenocarcinomas and intraductal papillary mucinous neoplasms with moderate dysplasia
}

\author{
Jonathan R Brody ${ }^{1, *}$, Agnes Witkiewicz ${ }^{2, *}$, Timothy K Williams ${ }^{1}$, Shrihari S Kadkol ${ }^{3}$, \\ Joseph Cozzitorto ${ }^{1}$, Brandice Durkan ${ }^{1}$, Gary R Pasternack ${ }^{4}$ and Charles J Yeo ${ }^{1}$ \\ ${ }^{1}$ Department of Surgery, Thomas Jefferson University, Philadelphia, PA, USA; ${ }^{2}$ Department of Pathology, \\ Thomas Jefferson University, Philadelphia, PA, USA; ${ }^{3}$ Department of Pathology, University of Illinois, \\ Chicago, IL, USA and ${ }^{4}$ Department of Pathology, Johns Hopkins University, Baltimore, MD, USA
}

\begin{abstract}
Nuclear phosphoprotein 32 (pp32) inhibits K-ras induced transformation in experimental models. pp32 mRNA expression correlates with differentiation status in breast and prostate cancers. In this study, we evaluated pp32 protein expression in relation to the differentiation status of pancreatic ductal adenocarcinomas and precursor lesions of the pancreatic cancers. pp32 expression showed strong nuclear staining in normal pancreatic acini and ducts. The intensity of this staining was maintained in pancreatic intraepithelial neoplasia, intraductal papillary mucinous neoplasms with mild dysplasia, well-differentiated adenocarcinomas, and in a subset of moderately differentiated adenocarcinomas. pp32 staining was absent or reduced in poorly differentiated tumors and in intraductal papillary mucinous neoplasms with moderate dysplasia. We validated pp32 expression by a second technique, immunoblot analysis of lysates from resected pancreatic ductal adenocarcinomas and pancreatic cancer cell lines. The well-differentiated pancreatic cancer cell line HPAC expressed high amounts of pp32, as compared to the poorly differentiated pancreatic cancer cell lines MiaPaCa2, PI19, and PI21 cells. Artificial introduction of pp32 expression into a poorly differentiated cell line, MiaPaCa2, caused an increase in G1 arrest compared to control cells. On the basis of this study and previous functional work that shows pp32 can inhibit K-ras transformation, we propose that reduction in pp32 expression levels may be a critical event in the progression of pancreatic tumorigenesis in an aggressive subset of pancreatic ductal adenocarcinomas.
\end{abstract}

Modern Pathology (2007) 20, 1238-1244; doi:10.1038/modpathol.3800974; published online 28 September 2007

Keywords: pancreatic ductal adenocarcinoma; pp32 (ANP32A); protein expression; tumor suppressor; poorly differentiated pancreatic adenocarcinomas; intraductal papillary mucinous neoplasms

Little is known about the molecular basis of poorly and undifferentiated pancreatic ductal adenocarcinomas, the most aggressive type of pancreatic cancer. We studied pp32 (also known as ANP32A) protein expression in pancreatic cancers because it has been shown to directly relate to the differentiation status of cancers in other systems and because previous functional work predicts a connection of pp32 to pancreatic tumorigenesis. ${ }^{1,2}$ pp32 is a

Correspondence: Dr JR Brody, PhD, Department of Surgery, Thomas Jefferson University, 611A Curtis, 1015 Walnut Street, Philadelphia, PA 19107, USA.

E-mail: jonathan.brody@jefferson.edu

*These two authors have contributed equally to this work.

Received 12 June 2007; revised and accepted 6 September 2007; published online 28 September 2007 multifunctional nuclear phosphoprotein that inhibits oncogene-mediated transformation in cell culture. $^{3-5}$ In relation to specific molecular aspects of pancreatic tumorigenesis, pp32 has been shown to inhibit a $K$-ras activating mutation, found in over $90 \%$ of pancreatic ductal adenocarcinomas, in both in vitro and in vivo models. ${ }^{3,5}$ Specifically K-ras dependent tumor formation in vivo is inhibited by pp32 overexpression and accelerated when pp32 expression is ablated. ${ }^{5}$ Moreover, pp32 was shown to bind to the phosphorylated form of $\mathrm{Rb}$ which is the dysregulated form of $\mathrm{Rb}$ found in nearly all pancreatic cancers due to p16 inactivation. ${ }^{6,7}$

Other pp32 functions related to tumorigenesis have been described and may explain why pp32 can inhibit K-ras signaling. pp32 has been shown to cooperate with Hur in stabilizing critical tran- 
scripts. ${ }^{8}$ It is believed that Hur plays a critical role in tumorigenesis. ${ }^{9}$ Further, pp32 has also been shown to form a complex that inhibits histone acetylation as part of the INhibitor of Histone Acetyl Transferase (INHAT) complex, and thus pp32 may help to regulate or repress transcriptional events critical for proliferation and tumorigenesis. ${ }^{10,11}$

pp32 mRNA expression has been studied in other epithelial tumor systems. In benign prostatic tissues, moderate pp32-related mRNA expression occurs only in the basal cells. ${ }^{1,12}$ A study of prostatic adenocarcinomas found elevated pp32 expression in $98 \%(54 / 55)$ of adenocarcinomas of Gleason score $\geq 5 \quad(P<0.0001) .{ }^{1}$ A follow-up study investigated pp32-related mRNA expression in human breast cancer. In breast cancer, abundant pp32-related mRNA expression was found in benign ducts and acini and in infiltrating ductal carcinomas. A total of $100 / 102$ cases were positive for pp32 expression. ${ }^{2}$ pp32's role in differentiation was functionally established when it was shown that long-term manipulated reduction of pp32 expression in the bladder carcinoma cell line, TSU-Pr1, induced neuronal differentiation. ${ }^{13}$

Taken together, pp32 expression appears to be a critical molecular marker and direct determinant of the state of differentiation in epithelial cancers. To date, pp32 expression has not been studied in normal pancreas and pancreatic ductal adenocarcinomas. The goal of this study was to correlate the differentiation status of pancreatic ductal adenocarcinomas with pp32 protein expression and to study the effect of manipulating pp32 expression in poorly differentiated pancreatic cancer cells.

\section{Materials and Methods}

\section{Specimens}

Thirty-five cases of pancreatic adenocarcinomas, five cases of pancreatic intraepithelial neoplasia and four cases of intraductal papillary mucinous neoplasms were retrieved from the Thomas Jefferson University pathology archives. The adenocarcinoma cases included 10 well, 15 moderately, and 10 poorly differentiated tumors. For three tumors, lymph node metastases were included in the analysis. Two pancreatic intraepithelial neoplasia1, three pancreatic intraepithelial neoplasia-3, and four intraductal papillary mucinous neoplasms (two with mild and two with moderate dysplasia) were also examined. This study was approved by the Institutional Review Board of the Thomas Jefferson University.

\section{Immunohistochemistry}

Formalin-fixed, paraffin-embedded blocks were processed for immunohistochemical analysis using heat antigen retrieval and avidin-biotin complex technique. pp32 antibody was applied to slides and incubated for $60 \mathrm{~min}$ using a 1/200 dilution (see description of the pp32 antibody in the next section). Staining intensity was scored blindly as strong, weak, or negative on the basis of nuclear staining. Weak cytoplasmic staining or reactivity in rare cells $(<5 \%)$ was interpreted as negative. The staining pattern was scored as diffuse or focal based on the percentage of tumor cells staining ( $>70 \mathrm{vs}$ $5-70 \%$, respectively).

\section{Immunoblot Analysis}

Cells were lysed with an SDS-lysis buffer. A measure of 5-20 $\mu \mathrm{g}$ of protein per well was separated by electrophoresis for over $2 \mathrm{~h}$ at $200 \mathrm{~V}$ using $10 \%$ bis-tris-polyacrylamide gels (Invitrogen, Carlsbad, CA, USA). After electrophoretic transfer of proteins on to polyvinylidene difluoride membranes, they were sequentially blocked in Tris-buffered saline/ $0.5 \%$ Tween (TBS-T) $/ 5 \%$ milk, incubated with primary pp32 antibody (1:1000) and then incubated with a horseradish peroxidase-conjugated secondary antibody (1:20 000, GE Healthcare, Piscataway, NJ, USA). ${ }^{4}$ The production of pp32 antibody was described previously. ${ }^{4,14}$ The protein bands were visualized on X-ray film using the Immobilon Western Chemiluminescent Substrate (Millipore, Billerica, MA, USA). Fast Green stain was used to stain the membrane to validate equal protein loaded per well (USB, Cleveland, OH, USA). At least two experiments were performed for each data point, and representative data are shown.

\section{Cancer Cell Lines}

Cancer cell lines were maintained in DMEM (low glucose, Invitrogen) containing 10\% FBS, 1\% pen./ strep., and 1\% L-glutamine. Pl19 and Pl21 cell lines were donated to us by Dr Elizabeth Jaffee (Johns Hopkins University, Baltimore, MD, USA). All other pancreatic cancer cell lines (MiaPaCa2, HPAC, XPA1, and Su86.86) were donated by Dr Scott Kern (Johns Hopkins University, Baltimore, MD, USA).

\section{Transfection of Pancreatic Cancer Cells}

The pp32 cDNA sequence was cloned into the pcDNA3.1Zeo + vector (Invitrogen) to obtain optimal expression post transfection. Stable transfection of MiaPaCa2 cells were performed using Lipofectin ${ }^{\mathrm{TM}}$ reagent (Invitrogen). Equal cells were plated in T-75 flasks and were transfected at $40-50 \%$ confluency using $10 \mu \mathrm{g}$ of 32.CMV (sense-pp32 construct) or EV.CMV (empty vector). In brief, cells were maintained in a transfection mix with Lipofectin reagent for $5 \mathrm{~h}$ and then replenished with standard DMEM high glucose media with $10 \%$ FBS and 1\% pen./ strep. and were allowed to recover for $72 \mathrm{~h}$. Cells 
were then trypsinized and replated in equal numbers into six-well plates and T-75 plates and the selection was done with $50 \mu \mathrm{g} / \mathrm{ml}$ of zeocin, as determined by an antibiotic kill curve. Selection took place over several weeks to allow for the plasmids to integrate into the cells.

\section{Cell Cycle Studies}

Overexpressing pp32 MiaPaCa2 cells (Mia.32) and empty vector stably transfected cells (Mia.EV) were plated equally into T-75 flasks and grown to confluency. Adherent cells were trypsinized and collected for staining using propidium iodide (PI). In brief, cells were fixed in ethanol and placed in a PI-staining solution containing $20 \mu \mathrm{g} / \mathrm{ml}$ PI, $100 \mu \mathrm{g} /$ $\mathrm{ml}$ RNase A, and $0.1 \%$ Triton X-100 (Sigma, St Louis, MO, USA). Cell cycle analysis was subsequently performed using flow cytometry and analyzed with the System II software (Beckman, Fullerton, CA, USA).

\section{Results}

\section{pp32-Specific Expression in Pancreatic Tissue}

By immunohistochemistry pp32 exhibited strong nuclear staining in normal pancreatic acini and ducts (Figure 1a). Variable nuclear reactivity for pp32 was identified in all cases of pancreatic ductal adenocarcinomas. Ten well differentiated and eight moderately differentiated adenocarcinomas showed diffuse, strong nuclear staining of the intensity equal to normal pancreatic tissue (Figure 1b). In contrast, nine cases of poorly differentiated and seven cases of moderately differentiated adenocarcinoma exhibited diffuse to focal weak staining (Figure 1c). Markedly anaplastic nuclei were often negative for pp32 expression. The intensity of staining in metastatic carcinoma in lymph nodes was focal and weaker when compared to the corresponding primary tumor (Figure 1d). The results of immunohistochemical staining are summarized in Table 1.

A unique pattern of pp32 expression was observed in intraductal papillary mucinous
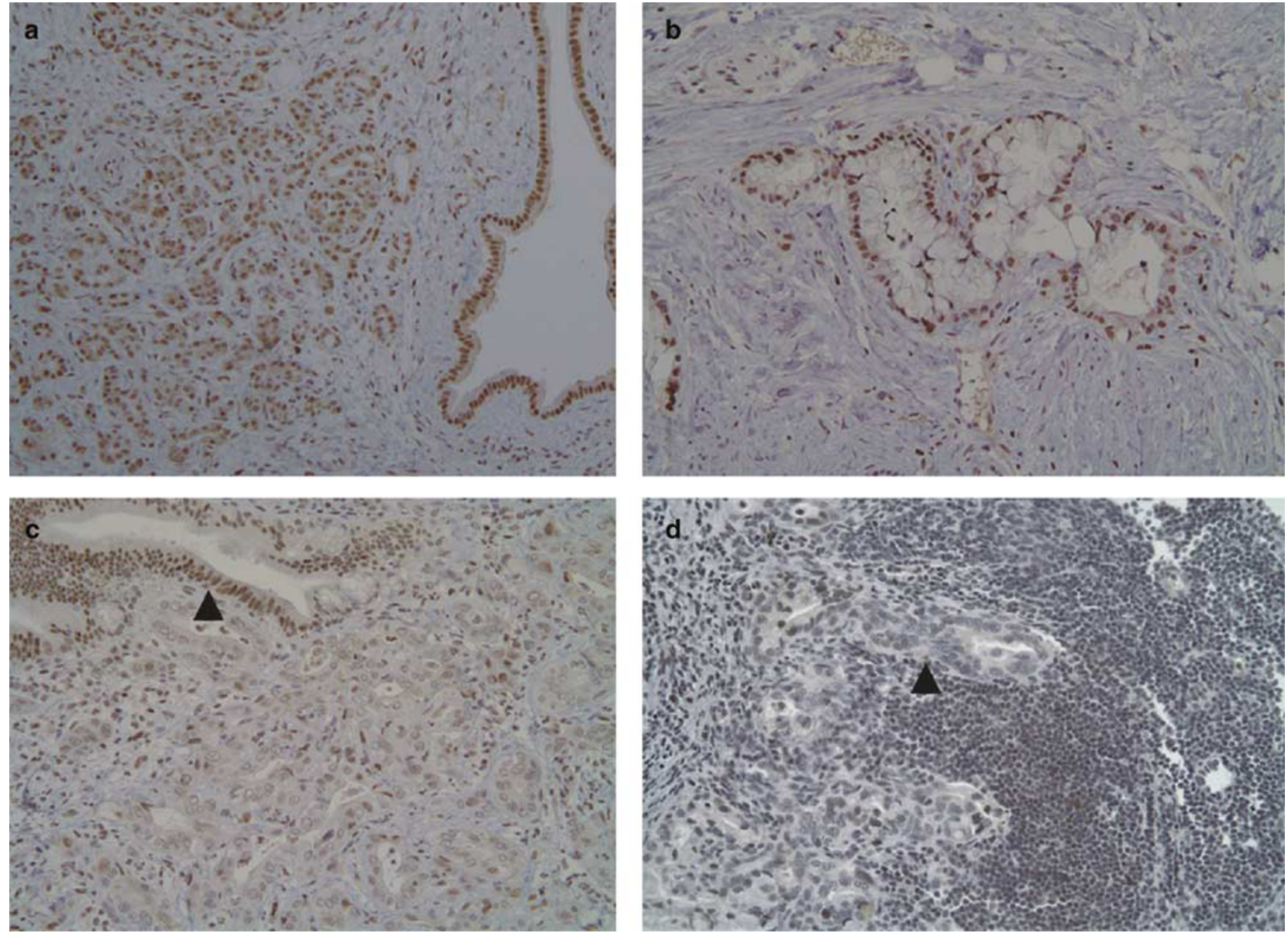

Figure 1 Strong nuclear expression of pp32 in (a) normal pancreatic acini and ducts and (b) in well-differentiated adenocarcinoma. (c) Poorly differentiated adenocarcinoma showing weak pp32 staining; note strong expression of pp32 in normal pancreatic duct (arrowhead). (d) Metastatic adenocarcinoma to lymph node (arrowhead) showing weak to absent staining for pp32 (for all images $\times 200$, stained for pp32). 
Table 1 Summary of pp32 expression in precursor pancreatic cancerous lesions and pancreatic ductal adenocarcinomas resected and stained with anti-pp32 antibody

\begin{tabular}{|c|c|c|c|c|c|c|}
\hline & $\begin{array}{c}\text { Intraductal } \\
\text { papillary mucinous } \\
\text { neoplasms } \\
\mathrm{n}=(4)\end{array}$ & $\begin{array}{l}\text { Pancreatic } \\
\text { intraepithelial } \\
\text { neoplasia } \\
\mathrm{n}=(5)\end{array}$ & $\begin{array}{c}\text { Well differentiated } \\
\text { adenocarcinoma } \\
\mathrm{n}=(10)\end{array}$ & $\begin{array}{c}\text { Moderately } \\
\text { differentiated } \\
\text { adenocarcinoma } \\
\mathrm{n}=(15)\end{array}$ & $\begin{array}{c}\text { Poorly differentiated } \\
\text { adenocarcinoma } \\
\mathrm{n}=(10)\end{array}$ & $\begin{array}{l}\text { Lymph node } \\
\text { metastasis } \\
\mathrm{n}=(3)\end{array}$ \\
\hline $\begin{array}{l}\text { Strong, diffuse } \\
\text { expression }\end{array}$ & $2 / 4$ & $5 / 5$ & $10 / 10$ & $8 / 15$ & $1 / 10$ & $0 / 3$ \\
\hline $\begin{array}{l}\text { Weak, diffuse, or } \\
\text { focal expression }\end{array}$ & $2 / 4$ & $0 / 5$ & $0 / 10$ & $7 / 15$ & $9 / 10$ & $3 / 3$ \\
\hline
\end{tabular}
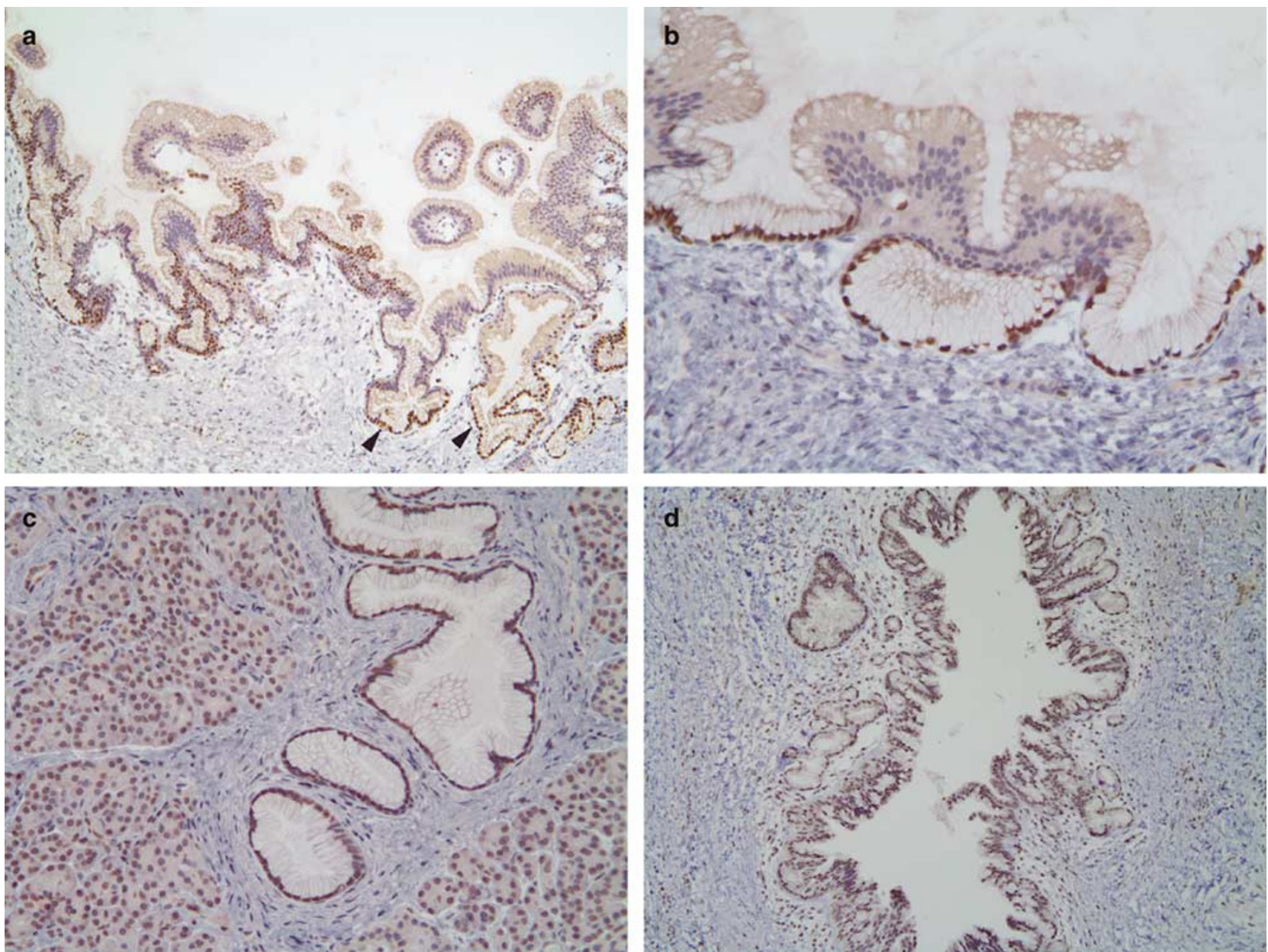

Figure 2 pp32 expression in precursor pancreatic ductal adenocarcinoma lesions. (a) Low-power magnification showing pp32 staining in intraductal papillary mucinous neoplasms with mild (arrowheads) to moderate dysplasia $(\times 100)$. (b) Strong nuclear staining for pp32 in basally located nuclei of cells with mild dysplasia and loss of nuclear stain in stratified cells of moderate dysplasia $(\times 200)$. (c) Strong nuclear staining for pp32 in pancreatic intraepithelial neoplasia-1 lesions. (d) Pancreatic intraepithelial neoplasia-3 lesions $(\times 200)$.

neoplasms. Areas of low-grade dysplasia showed strong nuclear staining in basally located nuclei, whereas areas of moderate dysplasia showed loss of nuclear staining and faint cytoplasmic positivity (Figure 2a and b). We observed pp32 specific staining in pancreatic intraepithelial neoplasia lesions independent of degree of the dysplasia (Figure 2c and d).

\section{Analysis of pp32 Protein Expression by Immunoblot}

Three out of three well to moderately differentiated pancreatic ductal adenocarcinomas showed pp32 expression while none of the poorly differentiated pancreatic ductal adenocarcinomas showed pp32 expression by immunoblot (Figure 3a). We then analyzed five pancreatic cell lines for pp32 


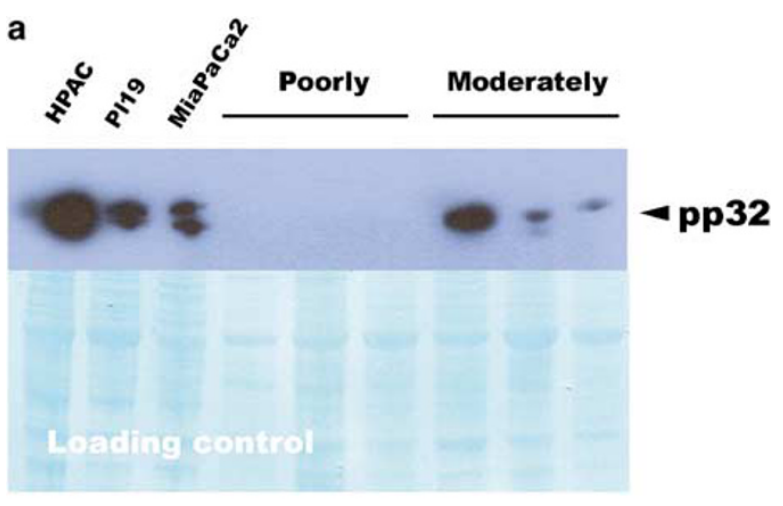

b

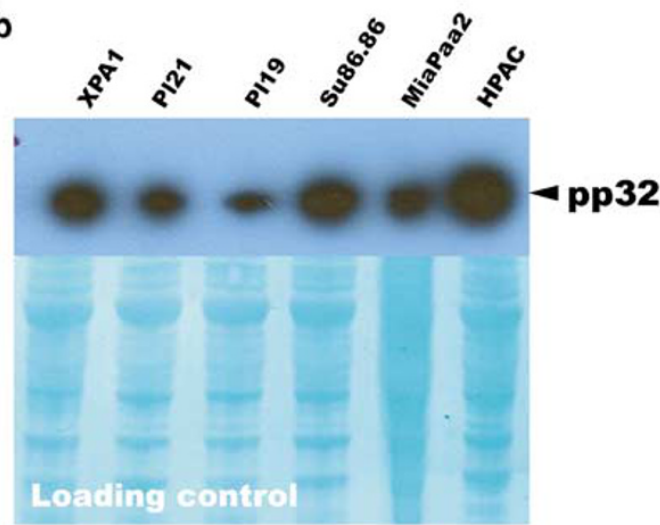

Figure 3 Immunoblot analysis of pp32 protein expression. (a) pp32 expression is the highest in the well-differentiated pancreatic cell line, HPAC. Poorly differentiated pancreatic cell lines Pl19 and MiaPaCa2 express low pp32 protein compared to HPAC. Three out of three poorly differentiated resected pancreatic ductal adenocarcinomas showed no pp32 expression (including at high exposure) (data not shown). Three out of three moderately differentiated resected pancreatic ductal adenocarcinomas expressed pp32. (b) Survey of pancreatic cancer cell lines, the known differentiation status in parentheses (if known), XPA1, Pl21 (poorly), Pl19 (poorly), SU86.86, MiaPaCa2 (poorly), and HPAC (well differentiated). Equal cells or tumor tissue were loaded per lane. Loading control is Fast Green stain (USB) of total proteins from the same blot exposed in the figure. Note protein overload in (b) of MiaPaCa2.

expression. All pancreatic cancer cell lines expressed pp32 on immunoblot analysis (Figure 3a and b). A moderately to well-differentiated pancreatic cancer cell line, HPAC (www.ATCC.org), expressed the highest amount of pp32 protein. The poorly differentiated pancreatic cell lines, MiaPaCa2, Pl19, and Pl21, had at least a three-fold reduction in pp32 protein expression compared to HPAC (Figure 3).

\section{pp32 Overexpression in Poorly Differentiated Pancreatic Cancer Cells}

Constitutive, stable pp32 expression under the selection of zeocin and a strong CMV promoter were achieved in the MaiPaCa2 cell line (Figure 4a). After a month under zeocin selection, a unique cellular morphology was noted in a number of the Mia.32 clones compared to the Mia.EV control cells
(Figure 4b). A number of Mia.32 clones did not proliferate or survive after reaching a certain size in diameter. Since at this time point the zeocin-resistant plasmid (both pp32.CMV and EV.CMV) integrated into the cells, we deduced this phenotypic change was due to pp32 overexpression (Figure 4b). Of those clones that survived, we were able to maintain a pooled MiaPaCa2 cell line in long-term culture that overexpressed pp32 (Figure 4a). These surviving cells were used for cell cycle analysis (Figure 4c). Consistent with pp32 having tumor suppressor qualities, the Mia.32 line (described in the Materials and Methods section) had a greater percentage of cells arrested in $\mathrm{G}_{1} / \mathrm{G}_{0}$ (average $=65.5 \%$ ) as compared to the Mia.EV cells (average $=56.1 \%$ ). The Mia.32 cells also had a smaller percentage of cells in S phase compared to the control cells (Figure 4c). No significant difference was found in the $G_{2} / M$ phase of the cell cycle (Figure 4c).

\section{Discussion}

Forty percent of resected pancreatic ductal adenocarcinomas are poorly differentiated and patients with this grade disease have an average median survival of 13 months. ${ }^{15}$ Understanding the molecular aspects of this subset of pancreatic cancers would be highly informative for our understanding of pancreatic tumorigenesis and the aggressiveness of this disease. pp32 expression has previously been shown to correlate with the differentiation status in breast and prostate cancers. ${ }^{1,2}$ In this study, we found that pp32 expression and intensity correlated with the differentiation status of pancreatic ductal adenocarcinomas (see Table 1). In general, welldifferentiated pancreatic ductal adenocarcinomas and pancreatic intraepithelial neoplasia lesions showed high expression levels of pp32; while poorly differentiated lesions had reduced expression. Similarly, intraductal papillary mucinous neoplasms with low-grade dysplasia showed strong, nuclear expression of pp32. However, there was a loss of pp32 staining in intraductal papillary mucinous neoplasms with moderate dysplasia. The later finding may reflect a higher malignant potential of intraductal papillary mucinous neoplasms versus pancreatic intraepithelial neoplasia lesions. Furthermore, these areas of moderate dysplasia may represent the first signs of progression toward malignant transformation. Future work will define whether loss of pp32 nuclear staining is a critical event in the sequence from intraductal papillary mucinous neoplasms with moderate dysplasia to intraductal papillary mucinous neoplasms with severe dysplasia/carcinoma in situ and invasive carcinoma. $^{16}$

The most intriguing and promising possibility for the reduction of pp32 expression in poorly differentiated pancreatic ductal adenocarcinomas and intraductal papillary mucinous neoplasms is 


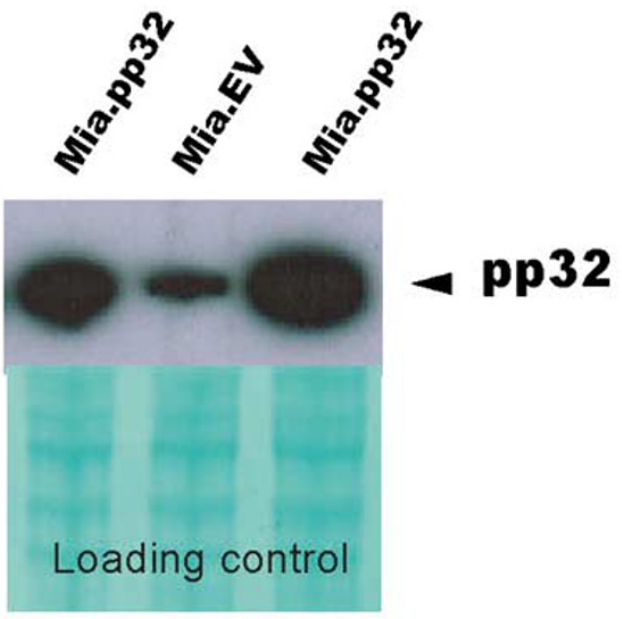

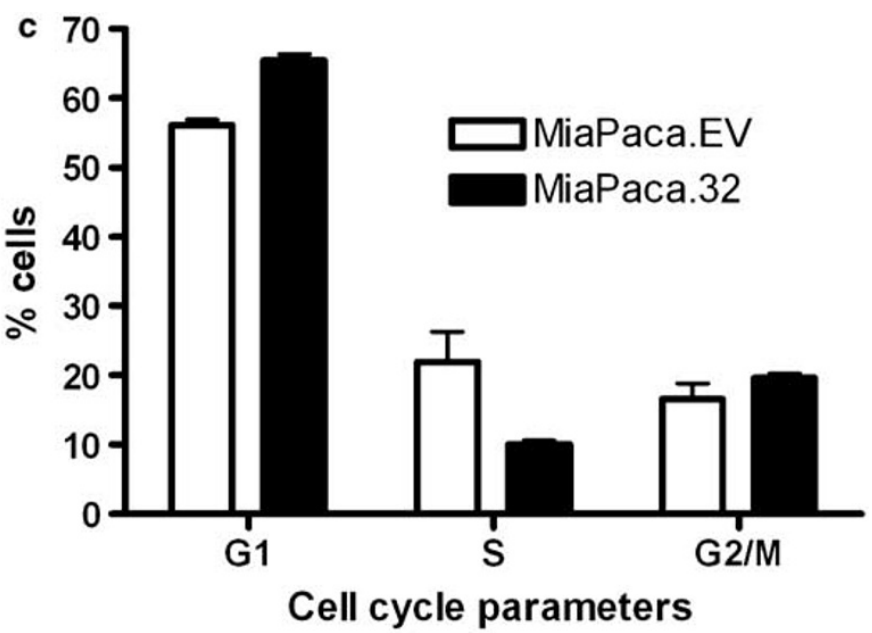

b

Mia.32 post-zeocin selection
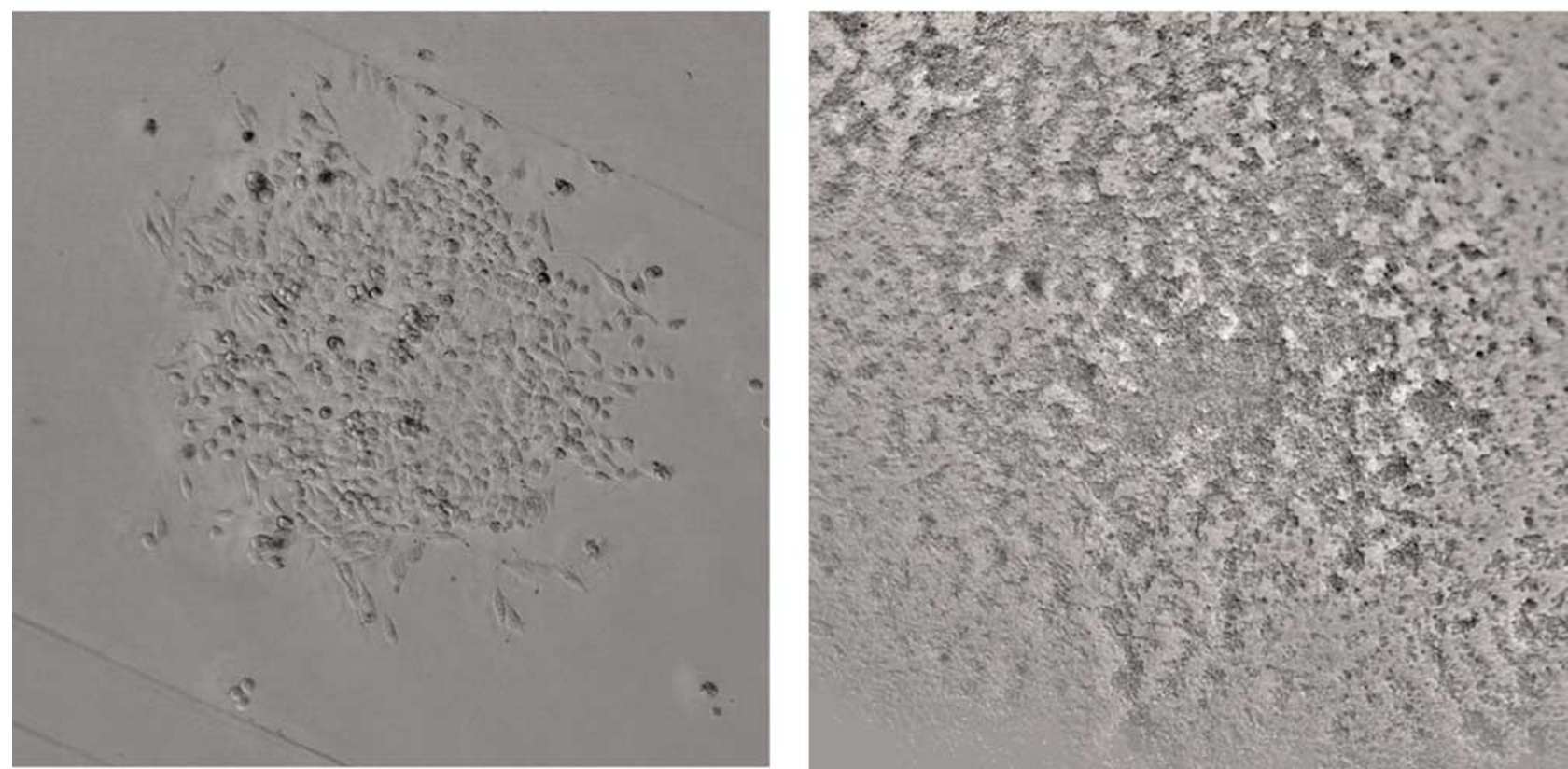

Figure 4 Introduction of pp32 expression into a poorly differentiated pancreatic cancer cell line, MiaPaCa2. (a) Representative lysates of MiaPaCa2 cells (Mia.32) stably transfected with the pp32.CMV vector run on a immunoblot for detection of pp32 protein expression. Control cells are the empty vector stable transfectants (labeled Mia.EV). Loading control is a Fast Green stain of the membrane. (b) Representative pictures from a confocal microscope of pp32 overexpressing clones after zeocin selection $(\times 10)$. The left panel represents a small Mia.32 clone and the right panel represents large Mia.32 clone. (c) Percent of Mia.32 and Mia.EV in different phases of the cell cycle. Mia.32 cells (average: $\mathrm{G}_{1} / \mathrm{G}_{0}, 65.5 \% ; \mathrm{S}, 9.99 \%$, and $\mathrm{G}_{2} / \mathrm{M}, 19.6 \%$ ); Mia.EV (average: $\mathrm{G}_{1} / \mathrm{G}_{0}, 56.1 \% ; \mathrm{S}, 21.95 \%$, and $\mathrm{G}_{2} / \mathrm{M}$, $16.6 \%$ ). Error bars represent s.d. of the mean, $n=2$.

pp32's ability to inhibit K-ras-induced transformation. $^{3-5}$ Since the majority of pancreatic ductal adenocarcinomas have an activating $K$-ras mutation and intraductal papillary mucinous neoplasms with moderate dysplasia have a greater likelihood to harbor K-ras mutations compared to intraductal papillary mucinous neoplasms with mild dysplasia, ${ }^{17}$ loss of pp32 staining in intraductal papillary mucinous neoplasms with moderate dysplasia may, in theory, aid the progress of these lesions to invasive carcinoma.
Previous work showed that pp32 overexpression negated K-ras-dependent tumor formation in mice and also inhibited $K$-ras mRNA expression. Moreover, ablation of pp32 expression accelerated K-ras dependent tumor formation and increased K-ras expression. ${ }^{5}$ In cell culture models, it was shown that pp32 could inhibit K-ras transformation in cooperation with a number of nuclear oncogenes including $c$-myc, $c$-jun, and E1A. ${ }^{4}$ This provides evidence that pp32 expression may be able to inhibit K-ras, dependent transformation in a 
heterogeneous genetic background found in most pancreatic tumors.

Introduction of pp32 expression into a poorly differentiated pancreatic cancer cell line (MiaPaCa2) that has a functional $K$-ras mutation underscored the importance of this molecule. Future studies will reveal the exact molecular mechanism in which pp32 expression arrested this poorly differentiated pancreatic cell line into the $G_{1} / G_{0}$ phase of the cell cycle and caused an initial phenotypic effect on cell survival (Figure 4). In brief, expression of pp32 in MiaPaCa2 cells may have regulated: (1) transcriptional networks important for proliferation and cell survival as part of the INHAT complex; ${ }^{11,13}$ (2) mRNA stability of important transcripts through its interaction with Hur, ${ }^{8,9}$ and (3) pp32's ability to bind to the phosphorylated form of $\mathrm{Rb}^{6}{ }^{6}$

Together these findings suggest that a change in pp32 expression levels may be an important contributor to tumorigenesis and thus a plausible therapeutic target in poorly differentiated pancreatic ductal adenocarcinomas. It is intriguing to hypothesize that pp32 upregulation in poorly differentiated pancreatic ductal adenocarcinomas may directly signal cancer cells to behave less aggressively. ${ }^{5,13}$ Studies correlating pp32 status with clinical outcome and exploring the role of this multidimensional molecule in pancreatic tumorigenesis are currently ongoing.

\section{References}

1 Kadkol SS, Brody JR, Epstein JI, et al. Novel nuclear phosphoprotein pp32 is highly expressed in intermediate- and high-grade prostate cancer. Prostate 1998;34:231-237.

2 Kadkol SS, El Naga GA, Brody JR, et al. Expression of pp32 gene family members in breast cancer. Breast Cancer Res Treat 2001;68:65-73.

3 Brody JR, Kadkol SS, Mahmoud MA, et al. Identification of sequences required for inhibition of oncogenemediated transformation by pp32. J Biol Chem 1999;274: 20053-20055.

4 Chen TH, Brody JR, Romantsev FE, et al. Structure of pp32, an acidic nuclear protein which inhibits onco- gene-induced formation of transformed foci. Mol Biol Cell 1996;7:2045-2056.

5 Bai J, Brody JR, Kadkol SS, et al. Tumor suppression and potentiation by manipulation of pp32 expression. Oncogene 2001;20:2153-2160.

6 Adegbola O, Pasternack GR. Phosphorylated retinoblastoma protein complexes with pp32 and inhibits pp32-mediated apoptosis. J Biol Chem 2005;280: 15497-15502.

7 Schutte M, Hruban RH, Geradts J, et al. Abrogation of the $\mathrm{Rb} / \mathrm{p} 16$ tumor-suppressive pathway in virtually all pancreatic carcinomas. Cancer Res 1997;57:3126-3130.

8 Brennan CM, Gallouzi IE, Steitz JA. Protein ligands to HuR modulate its interaction with target mRNAs in vivo. J Cell Biol 2000;151:1-14.

9 Lopez de Silanes I, Lal A, Gorospe M. HuR: posttranscriptional paths to malignancy. RNA Biol 2005;2: 11-13.

10 Fan Z, Zhang H, Zhang Q. Tumor suppressor pp32 represses cell growth through inhibition of transcription by blocking acetylation and phosphorylation of histone $\mathrm{H} 3$ and initiating its proapoptotic activity. Cell Death Differ 2006;13:1485-1494.

11 Seo SB, Macfarlan T, McNamara P, et al. Regulation of histone acetylation and transcription by nuclear protein pp32, a subunit of the INHAT complex. J Biol Chem 2002;277:14005-14010.

12 Kadkol SS, Brody JR, Pevsner J, et al. Modulation of oncogenic potential by alternative gene use in human prostate cancer. Nat Med 1999;5:275-279.

13 Brody JR, Kadkol SS, Hauer MC, et al. pp32 reduction induces differentiation of TSU-Pr1 cells. Am J Pathol 2004;164:273-283.

14 Malek SN, Katumuluwa AI, Pasternack GR. Identification and preliminary characterization of two related proliferation-associated nuclear phosphoproteins. J Biol Chem 1990;265:13400-13409.

15 Winter JM, Cameron JL, Campbell KA, et al. 1423 pancreaticoduodenectomies for pancreatic cancer: a single-institution experience. J Gastrointest Surg 2006; 10:1199-1211.

16 Feldmann G, Beaty R, Hruban RH, et al. Molecular genetics of pancreatic intraepithelial neoplasia. J Hepatobiliary Pancreat Surg 2007;14:224-232.

17 Z'graggen K, Rivera JA, Compton CC, et al. Prevalence of activating K-ras mutations in the evolutionary stages of neoplasia in intraductal papillary mucinous tumors of the pancreas. Ann Surg 1997;226:491-498; discussion 498-500. 